\title{
Discussion on the construction of shared management platform for large instrument in University
}

\author{
Yanping Zhu \\ Jilin Agricultural University, Changchun 130118, China
}

\begin{abstract}
Based on the modern management concept and the rapid development of information system, the university's large-scale instruments sharing platform is a powerful tool of the rational use of limited resources. The large-scale instruments sharing platform realizes the transition from conventional manual management to network information management. It provides detailed scientific basis for the lab's efficient macro control, construction and development. The construction experience of sharing platform and the reserve design of later individual requirements can afford lessons for the construction of sharing platform in other various fields.
\end{abstract}

Keywords: universities; large-scale instruments; sharing platform; information construction.

\section{Introduction}

Large equipment is an important material basis for the high level of school teaching and research university personnel training and an important guarantee for scientific research, it is subject platform construction and technical support of the core, but also society's scarce resources. Currently the number of large-scale equipment of the university on the one hand, more and more limited investment in education cannot meet the growing demand for social sun and the moon; the other hand, the existing benefits of large-scale equipment is not fully [1].

Due to the amount of research activities resulting device could not fully utilized, some large precision instruments inactivity [2]. All along, to meet the specific research, or other specialized departments operate autonomously internal demand, how to play a good opening, platform sharing, public support and other common needs, are universities and research institutes laboratory equipment management problems. The reason is the management concept, problem sharing and information management means of [3]. The traditional device management is static, the information lag, and lack effective laboratory open around the clock technical support [4]. Establishment of a modern large-scale equipment sharing platform, rational and effective use of large precision instruments and equipment resources, to achieve high-quality resource sharing, improve the utilization of equipment, so that large-scale precision instruments and equipment play a greater role in teaching, scientific research and technological innovation [3]. Large instruments sharing system is to promote large scientific instruments sharing of resources, improve the utilization of equipment, to achieve optimal allocation of resources and the construction of an open technology infrastructure. Through the construction of large-scale equipment sharing platform of information systems for users to set up information exchange platform, and gradually realize the dynamic management of large-scale information technology equipment and development instruments.

By integrating university equipment resources, will be scattered in various departments of the university departments of large scientific instruments optimized restructuring, school and community-oriented open source, efficient use of scientific instruments resources, avoid duplication and waste purchase, and ultimately a limited funding and resources for more scientific research personnel services.

\section{Method}

University's open sharing platform is the best instrument by the faculties of two stages, through the form of a network of schools for the sharing of large instruments and equipment to share a unified platform to achieve centralized management of decentralized virtual device [4]. Reception centers are university information portal School colonel large external display window of the instrument 
information platform, this service can dynamically display platform large display instrument status quo equipment resources school, secondary colleges internet link, the reservation process information, equipment distribution and electronic map display the instrument classified information, hot instrument display, the latest appointments, equipment operation and gig information, instrument monthly/annual statistics and other information when the machine is to provide internal and external users, service feedback research institutes and enterprises and between the school management interactive information exchange platform.

Two platforms - the hospital center stage center platform bear specific business processes through information exchange center to further demonstrate the Center's press release, equipment resources, equipment reservation information, publishing instrument training programs. Showcase the latest schedule information and real-time instruments experimental use of information, the use of instruments and equipment movements, instrument cumulative monthly / annual ranking, the latest gigs and public data storage.

Test Center is a large scientific instrument and equipment composed of Science and Technology Platform, Science and Technology Platform is the most important and most critical, most high-end platform. Test Center "Scientific Management" construction mode for the college major instrument to create a public platform for building good start, so that large equipment resource sharing is possible, truly shared, to have modern management tools to achieve electronic information equipment, information technology systems and processes will be convenient to use an appointment, teachers and students and other users will be able to find a one-stop service they need large-scale equipment, to make a reservation of the instrument, the use of more equitable and transparent. Establish a unified archiving, management and use patterns, to ensure data harmonization and standardization.

Establish a sound and effective functioning of the shared platform should be included the following modules. Including: 1, implementation platform from a traditional manual management to rely on a network of information management; 2, unified authentication, sharing and circulation of information and unified authentication data, student information synchronized with each other, staff and school user information, arranging information, student enrollment, laboratory equipment, information and other data, data exchange, data within the circulation system between systems; 3 , appointment management; 4 fund management, equipment management subsystem fee is set according to booking reservation management apparatus and instruments to achieve billing functions of the system. Includes: The capital account, consumer records check, charges statistics, the cost of cleaning equipment, fees, charges confirmed, payment and other functions; 5, remote control, instrumentation software client administrators can login authorization, remote boot, shutdown, restart, etc. operating remote viewing and access switch, the switch for remote access, set password access control and switch status and other operations, and other administrators to view real-time dynamic laboratory remotely via the Internet (such as off-site, at home).

\section{Discussion}

For a long time, after some large equipment purchase, limited to the unit, the department use, forming a "organizational unit Individual Ownership" semi-closed form, the use of instruments and equipment to be confined within a specific range, leading to internal and external open low level of sharing, a serious impediment to equipment usage and improving efficiency. Some are designed for the high degree of specialization of certain businesses and the acquisition of research projects, narrow the scope of application, general poor equipment, long after the end of the subject to be idle [1]. Although large-scale resource sharing instrument concept has a certain basis of consensus, there have been a lot of policy and regulatory measures introduced, but the shared services did not achieve the desired results.

Large instrument effectiveness and the degree of shared formation is not high, the impact of the factors varies. Under the current management, management level too; responsibilities between levels is not clear; the subject of the managed property characteristic grasp inaccurate; lack of limited 
administrative allocation of resources, and therefore cannot be implemented for effective management and sharing of various factors, "semi-closed" mode operation of the instrument seem like a chronic disease permanently. Therefore, try to stage large-scale demand for integrated open sharing equipment and services, factors attribute to acquire equipment and instrument functions, applications, staffing, evaluation criteria and incentive system, etc., the first consideration is to seek innovative management model.

Large instrument conceived two shared platform integrates various factors, both the maximum extent embodies the advantages of centralized management and focus on investment, but also reflects the scientific management and coordination of the model, but also has a considerable degree of flexibility. The starting point is relatively high, a large amount of preparatory investment, its role and effectiveness has been fully revealed in the practice of some universities, but it needs to be further improved. In short, establish a good mechanism to run large-scale open sharing instrument is a long-term, arduous and complex systems engineering, construction equipment shared management platform is an innovation gives the meaning of work.

\section{Conclusion}

Large Instrument and open sharing platform is one of the school teaching, scientific research and construction of essential public service system, public services, process-oriented, rule-based, cost breakdown of; get real first-hand data for all levels of management, through in-depth analysis of the data, in order to amend the original management and inappropriate allocation of funds, to achieve efficient macro-control, to provide the basis for detailed laboratory construction and development planning, construction and management to avoid disjointed, effective use of school limited construction funds to improve teaching and research, and promote interdisciplinary integration, strengthen the cultivation of high-level innovative talents plays a vital role.

\section{References}

[1] Iandoli L. Building Consensus in On-Line Distributed Decision Making: Interaction, Aggregation and the Construction of Shared Knowledge [M]// Consensual Processes. Springer Berlin Heidelberg, 2011:339-355.

[2] Liu T, Cao P, ZHANG Xiao-liang, et al. Exploration of Construction of the Management Platform for Opening and Sharing of Large-scale Instruments[J]. Research \& Exploration in Laboratory, 2014.

[3] LAN M, YE Z, CHEN X. Exploration on Set-up of Shared Management Platform for Large Scale Instruments in College [J] [J]. Research and Exploration in Laboratory, 2009, 4: 087.

[4] Rong-Tai Y U. The Practice and Exploration on the Research Function of Large-scale Instruments in Colleges [J]. Journal of Jingdezhen College, 2014. 